\title{
Nanoparticle-based optical biosensors for the direct detection of organophosphate chemical warfare agents and pesticides
}

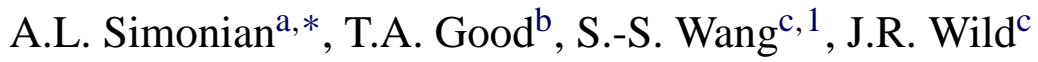 \\ a Materials Research and Education Center, Auburn University, 201 Ross Hall, Auburn, AL 36849, USA \\ ${ }^{\mathrm{b}}$ Chemical and Biochemical Engineering, UMBC, Baltimore, MD, USA \\ ${ }^{c}$ Biochemistry and Biophysics Department, Texas A\&M University, College Station, TX, USA
}

Received 21 March 2004; received in revised form 23 June 2004; accepted 23 June 2004

Available online 29 July 2004

\begin{abstract}
Neurotoxic organophosphates (OP) have found widespread use in the environment for insect control. In addition, there is the increasing threat of use of OP based chemical warfare agents in both ground based warfare and terrorist attacks. Together, these trends necessitate the development of simple and specific methods for discriminative detection of ultra low quantities of OP neurotoxins. In our previous investigations a new biosensor for the direct detection of organophosphorus neurotoxins was pioneered. In this system, the enzymatic hydrolysis of OP neurotoxins by organophosphate hydrolase $(\mathrm{OPH})$ generated two protons in each hydrolytic turnover through reactions in which $\mathrm{P}-\mathrm{X}$ bonds are cleaved. The sensitivity of this biosensor was limited due to the potentiometric method of detection. Recently, it was reported that a change in fluorescence properties of a fluorophore in the vicinity of gold nanoparticles might be used for detection of nanomolar concentrations of DNA oligonucleotides. The detection strategy was based on the fact that an enhancement or quenching of fluorescence intensity is a function of the distances between the gold nanoparticle and fluorophore. While these reports have demonstrated the use of nanoparticle-based sensors for the detection of target DNA, we observed that the specificity of enzyme-substrate interactions could be exploited in similar systems. To test the feasibility of this approach, OPH-gold nanoparticle conjugates were prepared, then incubated with a fluorescent enzyme inhibitor or decoy. The fluorescence intensity of the decoy was sensitive to the proximity of the gold nanoparticle, and thus could be used to indicate that the decoy was bound to the OPH. Then different paraoxon concentrations were introduced to the OPH-nanoparticle-conjugate-decoy mixtures, and normalized ratio of fluorescence intensities were measured. The greatest sensitivity to paraoxon was obtained when decoys and OPH-gold nanoparticle conjugates were present at near equimolar levels. The change in fluorescence intensity was correlated with concentration of paraoxon presented in the solution.
\end{abstract}

(C) 2004 Elsevier B.V. All rights reserved.

Keywords: Organophosphate hydrolase; Enzyme; Biosensor; Paraoxon; Direct detection; Gold nanoparticle; Fluorophore

\section{Introduction}

Organophosphorus (OP) neurotoxins comprise a unique class of contaminants and chemical warfare $(\mathrm{CW})$ agents that generally show low environmental persistence, but they have a high acute toxicity and a wide range of biological activities.

\footnotetext{
* Corresponding author. Tel.: +1 334844 4485; fax: +1 3348443400 .

E-mail address: als@eng.auburn.edu (A.L. Simonian).

1 Present address: Department of Chemical Engineering, National Taiwan University, Taipei, Taiwan.
}

Some members of this class are extremely toxic to mammals (e.g. the human oral lethal dose for paraoxon $=16 \mathrm{mg} / \mathrm{kg}$, and $\mathrm{VX}$ is lethal at $1 \mathrm{mg}$ if ingested or $100 \mathrm{mg} \mathrm{min} / \mathrm{m}^{3}$ if inhaled); these neurotoxins are powerful inhibitors of esterase enzymes, such as acetyl- and butyryl-cholinesterases or neurotoxic esterase, which are involved in nerve function [1]. The accurate detection of low concentrations organophosphate (OP) neurotoxins in environmental samples poses an extremely difficult challenge. Soil and water samples are very likely to contain organophosphate pesticides due to heavy urban and rural use of these compounds. Military and terroristic 
<smiles>CC(C)OP(=O)(F)OC(C)C</smiles>

DFP<smiles>CCOP(=O)(OCC)SCCSCC</smiles>

Demeton-S<smiles>CC(C)OP(C)(=O)F</smiles><smiles>CCOP(=O)(OCC)Oc1ccc([N+](=O)[O-])cc1</smiles>

Paraoxon

Fig. 1. Chemical structures of some organophosphate neurotoxins.

activities may result in air, water, and soil contamination with different chemical warfare $(\mathrm{CW})$ agents. The number of OP compounds that could be present in the environment continues to grow; during the past 50 years over 1500 neurotoxins, including new $\mathrm{V}$ and $\mathrm{G}$ types of chemical warfare agents whose chemical structures mimic those of much more environmentally tolerable pesticides, have been synthesized. The ability to discriminate between classes of OP neurotoxins is critical for the management of the impact of such supertoxicants because of the large number of different pesticides and $\mathrm{CW}$ agents in this class and their varying neurotoxicities. It is essential that $\mathrm{CW}$ agents can be readily and unequivocally distinguished from chemically similar agricultural compounds, and ubiquitous organophosphate pesticides must not appear as false positive indications of chemical warfare (CW) agents. Fig. 1 compares the chemical structure of five common organophosphate compounds; all are toxic cholinesterase inhibitors [2]. Paraoxon (PX), diisopropyl fluorophosphate (DFP), and demeton-S are commercially available organophosphate compounds.

Sensitive biosensors based on acetylcholinesterase (AChE) or butyryl cholinesterase (BChE) inhibition have been developed and used for OP agent detection [3-6]. A number of other enzymes such as urease and glucose oxidase have been also used in inhibition-based biosensors for OP neurotoxins $[7,8]$. In each of the inhibition-based biosensors, the OP agent interacts with the active site of the enzyme, resulting in loss of enzyme activity and hence a decrease in sensor signal. Inhibition-based sensors suffer from several limitations. First, any environmental or handling factors that cause loss of enzyme activity will result in false positive signals [9-11]. Second, such sensors require baseline testing prior to sample application and lengthy sample incubation times to allow enzyme-analyte interaction. Third, due to the irreversible nature of cholinesterase enzyme inhibition, inhibition-based sensors cannot be reused without regeneration of enzyme activity. Despite several attempts to improve the specificity of cholinesterase sensors by monitoring of inhibition with both butyryl and acetyl cholinesterase
[12] or analyzing inhibition patterns obtained with several acetyl cholinesterase enzymes from diverse sources [13], the inhibition-based sensors still have a number of unsolved problems.

The preferred strategy to overcome the disadvantages of inhibition-based sensors is the replacement of inhibition type recognition with catalytic recognition/utilization of the target agent. In this case fast enzyme kinetics is advantageous as it may be utilized for real time or near-real time analysis. In 1996, we pioneered the development of a new "catalytic" approach for direct detection of OP neurotoxins based on the enzyme organophosphate hydrolase [14], and further suggested a novel multi-enzyme strategy for discrimination between different classes of neurotoxins [9]. Our approach was based on organophosphorus hydrolase (OPH; E.C. 3.1.8.1), a well-characterized metalloenzyme originally isolated from Pseudomonas diminuta [15]. OPH exhibits the unique ability to hydrolyze a large variety of organophosphate pesticides and neurotoxins including paraoxon, parathion, acephate, Sarin, and VX [17]. The enzyme is capable of cleaving $\mathrm{P}-\mathrm{O}, \mathrm{P}-\mathrm{F}, \mathrm{P}-\mathrm{S}$, and $\mathrm{P}-\mathrm{CN}$ bonds via an $\mathrm{S}_{\mathrm{N}}$ 2-type mechanism, resulting in hydrolysis products, which change solution $\mathrm{pH}$ [16-18]. Direct neurotoxin detection is thus possible via measurement of the $\mathrm{pH}$ change associated with enzyme activity [14]; with $\mathrm{pH}$ measurements made either with conventional $\mathrm{pH}$ electrodes [14] or with $\mathrm{pH}$ sensitive fluorescent dyes [19]. This strategy can be extended to further discriminate between different classes of OP neurotoxins by monitoring additional organophosphate hydrolysis products. For example, hydrolysis of phosphofluoridates yields changes in $\mathrm{pF}$ as well as $\mathrm{pH}$ that can be detected with a fluoride specific ion-selective electrode [20]. While very sensitive and selective, such methods are highly dependent on the buffering capacity of the sample. Thus, new sensing technologies are needed which would complement the best of the existing technologies.

The present work describes a novel strategy for the direct detection of OP neurotoxins. Instead of using the $\mathrm{pH}$ change associated with enzymatic hydrolysis of the OP substrate as 
an indicator of the presence of an OP compound, the method described is based on the change in fluorescence of a competitive inhibitor of the OPH enzyme when the inhibitor is displaced by the OP substrate. The change in fluorescence of the inhibitor is produced by the presence of a gold nanoparticle attached to the enzyme.

Since mid-1980s, there have been reports of the unique optical and electronic properties of metal and semiconductor colloidal suspensions, especially when the particle size was at the nanoscale [21]. This large number of surface electrons gives rise to surface enhanced Raman and surface plasmon resonance effects that are highly dependent upon the size and degree of aggregation of the particles [22]. A variety of assays have been developed recently which exploit changes in optical properties in the vicinity of a either a gold or semiconductor nanosurface [23], including molecular beacons for the detection of DNA [24-26], surface plasmon resonance based assays [27], and surface enhanced resonance Raman assays [28]. Femtomolar concentrations of target DNA could be detected in these nanoparticle based detection systems [24]. These assays have been used in the detection of proteins including the estrogen receptor alpha [29], human serum albu$\min [30]$, wheat germ agglutinin, and epidermal growth factor [31]. The use of surface modified fluorescence has been reported recently for the detection of prostate specific antigens [32]. Antibodies and complementary DNAs have been used as the recognition element. However, to our knowledge, the work we present is the first to use an enzyme as the recognition element in a nanoparticle or surface modified fluorescence assay.

A variety of analytical techniques have been developed which exploit changes in fluorescence properties of a molecule in different environments, whether those changes are quenching [33], Förster resonance energy transfer [34], or surface modified fluorescence [35]. Molecular beacons provide an example of the use of surface modified fluorescence for the detection of DNA with sensitivity down to the mid nanomolar level [36]. While these reports have demonstrated the use of nanoparticle based sensors for the detection of target DNA, the specificity of enzyme-substrate interactions could easily be exploited in similar systems. The requirements for a successful sensor are: (1) high specificity in binding between recognition molecule and target, and (2) the ability to easily manipulate the distance between nanoparticle and fluorophore in the response to target molecule concentration. This leads us to believe that we should be able to develop a simple method for organophosphate neurotoxins detection that uses nanoparticle surface modified fluorescence based on highly specific recognition of OP by OPH enzyme.

A primary thrust of the present work is to develop, evaluate and demonstrate a new sensing approach based on a technology analogous to "molecular beacons". This involves the fluorescence modification of a signal from a specific decoy (or competitive inhibitor) as the result of the proximity to a nanoparticle surface upon which an enzyme-based biosensing element is conjugated.

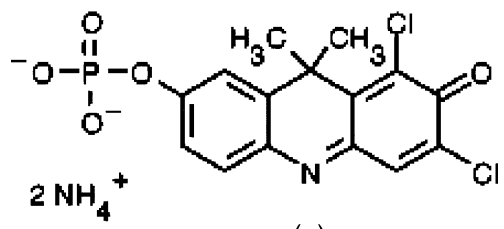

(a)<smiles>Cc1cc(=O)oc2c(F)c(OP(=O)(O)O)c(F)cc12</smiles>

(b)

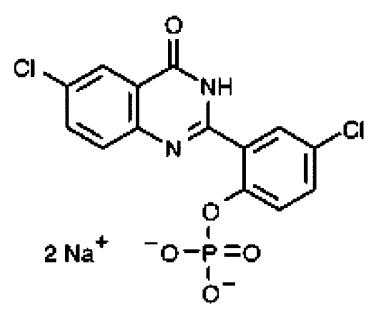

(c)

Fig. 2. Fluorophore structures: (a) 7-hydroxy-9H-(1,3-dichloro-9,9dimethylacridin-2-one (DDAO phosphate), (b) Difluorinated methylumbelliferyl phosphate (DiFMUP), and (c) ELF 97 phosphate.

\section{Experimental}

\subsection{Reagents and buffers}

Paraoxon (diethyl-p-nitrophenyl phosphate) and reagents for buffer (CHES (2-[cyclohexylamino] ethanesulfonic acid), $\mathrm{CoCl}_{2} \cdot 6 \mathrm{H}_{2} \mathrm{ONaCl}, \mathrm{MnCl}_{2}$ ) were obtained from Sigma Chemical Company (USA). 7-Hydroxy-9H-(1,3-dichloro9,9-dimethylacridin-2-one (DDAO phosphate), Difluorinated methylumbelliferyl phosphate (DiFMUP), and ELF 97 phosphate, were obtained from Molecular Probes. Monomaleimido Nanogold, and sulfo- $N$-hydroxy-succinimido Nanogold were purchase from Nanoprobes (Yaphank, NY, USA). The chemical structures of all fluorophore decoys are presented in Fig. 2 (from http://www.probes.com/servlets/ structure). All solutions were prepared using $18 \mathrm{M} \Omega \mathrm{cm}$ ultrapure water (Milli-Q Plus, Millipore, ST).

\subsection{Enzyme and nanoparticle/enzyme conjugates}

Native OPH (E.C. 3.1.8.1) was isolated from a recombinant Escherichia coli strain using published procedures [17]. The number of attachment sites for gold on the enzyme will affect the ability to precisely control the distance between nanogold and decoy, which, as stated earlier, will significantly affect the fluorescence modification properties of the sensor. A review of crystal structures of OPH [37] revealed 


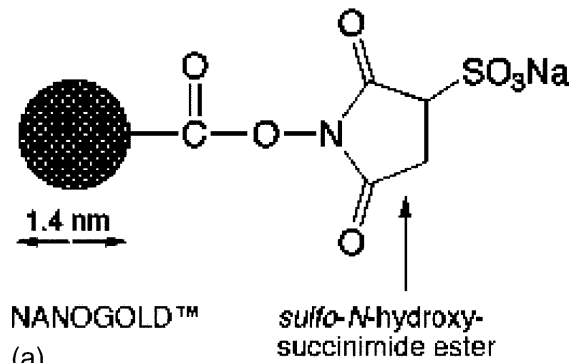

(a)

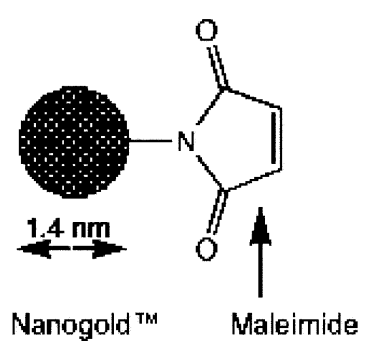

Fig. 3. Schematic showing a structure of Nanogold sulfo-succinimide group (a) and maleimide group (b) (http://www.nanoprobes.com/Inf2020.html).

six primary amines on each OPH monomer in the form of lysine residues, and only two sulfhydryl groups in the form of cysteine residues, on the OPH molecule. Thus, there are two different approaches that may be used to attach gold nanoparticles to OPH. Since the primary amines in lysine are targets of attachment for succinimidyl esters, gold nanoparticles will interact with the reactive sulfo- $N$-hydroxy succinimide functionality (Nanogold 1) (Fig. 3a) may be covalently linked to OPH lysine residues. Another approach is to use the Nanogold particle with a single maleimide functionality incorporated into a ligand on the surface of the gold particle (Nanogold 2) (Fig. 3b); this has a specific reactivity towards sulfhydryl groups and may be covalently linked to cysteine residues in $\mathrm{OPH}$.

To prepare OPH/mono-sulfo-NHS-nanogold conjugate, Nanogold reagent was dissolved in $1 \mathrm{ml}$ deionized water, and the protein $(1 \mathrm{mg} / \mathrm{ml}$ final concentration) was allowed to react with the Nanogold in buffer solution at $\mathrm{pH} 7.5-8$ overnight at $4{ }^{\circ} \mathrm{C}$. Sufficient reagent was supplied to label $6 \mathrm{nmol}$ of amine sites. Unbound Nanogold particles were removed by ultrafiltration, using Millipore tubes MICROCON YM-10 (MWCO 10,000) $3 \times$ at 14,000g $(12.3 \times 1000 \mathrm{rpm}$ on Eppendorf Centrifuge 5415C, $30 \mathrm{~min})$. The extent of labeling was calculated from the UV-vis spectrum of the conjugate according to the manufacturers' recommendations. Similar procedures were performed for preparation of the monomaleimido nanogold-OPH conjugate. Nanogold conjugates were stored in $0.02 \mathrm{M}$ sodium phosphate buffer with $150 \mathrm{mM}$ sodium chloride.

\subsection{Apparatus}

The enzymatic activity of $\mathrm{OPH}$ and $\mathrm{OPH} /$ conjugates were monitored with a UV-vis spectrophotometer "UltraSpec 2100pro" (Amersham Bioscience, USA) by titration with $2.5 \mathrm{mM}$ paraoxon while solutions were continuously stirred. The fluorescence measurements were performed using a QM-1 fluorescence spectrometer (Photon Technologies International, Monmouth, NJ). The excitation wavelengths for DDAO, DiFMUP and ELF 97 phosphate were 478, 360 and was $350 \mathrm{~nm}$, respectively; the paraoxon-induced changes in decoys emission intensity were monitored at $628 \mathrm{~nm}$ for DDAO, at $460 \mathrm{~nm}$ for DiFMUP, and $550 \mathrm{~nm}$ for ELF 97 phosphate.

\subsection{Detection procedure}

Prior to experimental analysis, a stock solution of DDAO phosphate fluorophore was prepared at concentrations of $10^{-6}$ to $3 \times 10^{-6} \mathrm{M}$ in DI $\mathrm{H}_{2} \mathrm{O}$. Fluorescence intensity $\left(\mathrm{IF}_{1}\right)$ of DDAO was measured and used as a background signal level. OPH/gold nanoparticle conjugate was added and intensity of fluorescence of the conjugate-decoy complex $\left(\mathrm{IF}_{2}\right)$ was measured again. Paraoxon was added in different concentrations and fluorescence intensities, $\left(\mathrm{IF}_{3}\right)$ were measured. Relative fluorescence intensity change $\Delta \mathrm{IF}$ was calculated as:

$\Delta \mathrm{IF}=\frac{\mathrm{IF}_{3}-\mathrm{IF}_{1}}{\mathrm{IF}_{2}-\mathrm{IF}_{1}}$

This represents the ratio of enhancement of fluorescence in the presence of paraoxon to the enhancement of fluorescence in the absence of paraoxon. Two different nanogolds (Nanogold 1 and 2) were evaluated. Control experiments were performed to check any fluorescence intensity changes in the absence of OPH/gold conjugate.

\section{Results and discussion}

\subsection{Principle of enzyme/nanoparticle sensing}

We examined the feasibility of developing an enzymebased sensor for the detection of OP compounds that used nanoparticle modified fluorescence of an inhibitor of the enzyme to generate the signal for the OP compound detection. The principle behind sensor operation is shown in Figs. 4 and 5. As seen in Fig. 4 gold nanoparticle is covalently bound to an enzyme molecule. A fluorophore decoy, being a weak competitive inhibitor of $\mathrm{OPH}$ with a similar chemical structure to the substrate (analyte of interest), is introduced to the solution and is bound to the OPH active site. If the gold particle attached via amino- or sulfhydrylgroups to the $\mathrm{OPH}$ is at the certain distance from the decoy (typically between about 10 and $40 \mathrm{~nm}$ ), enhancement of fluorescence will be observed. The lower the quantum efficiency of the fluorophore, the greater potential enhancement when the decoy is bound to the enzyme-gold complex. If the nanoparticle is at a distance of greater than about $40 \mathrm{~nm}$ from the fluorophore, then fluorescence will be unaffected by the presence of the gold, leading to a reduction in fluorescence 


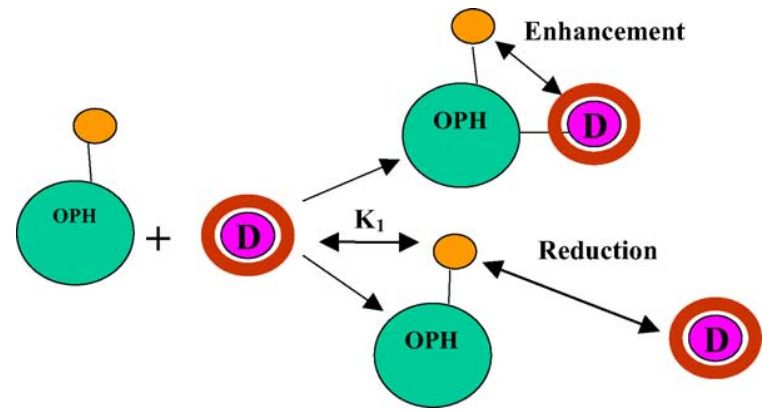

Fig. 4. Schematic of Decoy-Enzyme interaction for enhancement in the absence of substrate. Decoy (D) binds to enzyme-nanogold conjugate (OPH), leading to a surface enhanced fluorescence of the decoy.

signal. Once the decoy is bound to the OPH active site, then it is possible test for the presence of the analyte of interest (which is a substrate of OPH). If the substrate is present, then the analyte will displace the decoy because of its much higher affinity for the OPH active site, and the fluorescence signal of the sample will change. As seen in Fig. 5, for the case of an enhancement-based sensor, the analyte (indicated by S), will displace the decoy bound to the enzyme active site. As the decoy moves away from the gold nanoparticle, its fluorescence intensity will change. The change in fluorescence intensity is related to the concentration of analyte present in the solution.

\subsection{Kinetic evaluation of fluorescent molecules as possible OPH inhibitors}

Several fluorophore compounds were examined as possible candidates for the decoy or fluorescent inhibitor of $\mathrm{OPH}$, based on similarity of the chemical structures of the fluorophores (Fig. 2) with structures of OPH substrates (Fig. 1).

The catalytic rates $\left(k_{\text {cat }}\right)$ and dissociation constants $\left(K_{M}\right)$ for the enzyme in the presence and absence of the proposed inhibitors were determined by evaluating initial rates of enzyme reaction at constant enzyme concentration with variable concentrations of substrate (data are not presented). Based on a kinetic analysis of rates of enzymatic hydrolysis of paraoxon in the presence of DDAO, DiFMUP and ELF 97, it was observed that DDAO was a competitive inhibitor of $\mathrm{OPH}$, while DiFMUP and ELF 97 had no effect on enzymatic activity. These results suggested that DDAO was a suitable decoy for the proposed surface modified fluorescence method. Kinetic data indicated that the fluorophore binds to the active site

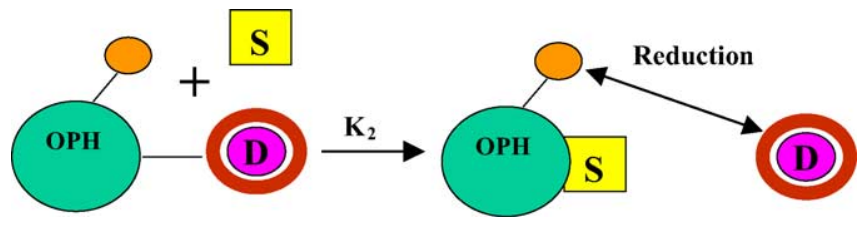

Fig. 5. Schematic of analyte (S) displacement of decoy (D) from OPH-gold complex $(\mathrm{OPH})$, leading to decrease fluorescence signal from the decoy. of $\mathrm{OPH}$ and competes with paraoxon, but has a dissociation constant $\left(K_{i}\right)$ that is several orders of magnitude smaller than the dissociation constant for paraoxon $\left(K_{m}\right)$ for OPH. Based on our data, $K_{m}$ and $K_{i}$ were on the order of $3 \times 10^{-5} \mathrm{M}$ and $10^{-7} \mathrm{M}$, respectively.

\subsection{Response on different paraoxon concentrations.}

Among the three fluorophores examined, only DDAO phosphate, a long-wavelength, dual-purpose fluorophore, exhibited the appropriate ability to change fluorescence intensity in response to different concentrations of paraoxon. Control experiments were performed to evaluate the influence of paraoxon on fluorescence intensity of DDAO in the absence of the enzyme, OPH (Fig. 6). There were only minor differences observed in fluorescence intensity of the decoy in the presence and absence of paraoxon when no enzyme-nanoparticle conjugate was present. Differences in fluorescence intensity between decoy and decoy plus paraoxon were buffer dependent and could be minimized by use of $20 \mathrm{mM}$ glycine buffer.

\subsection{Effect of different nanogold attachment chemistries}

Experiments were conducted using two different nanogold chemistries, Sulfo- $N$-hydroxy-succinimido Nanogold (Nanogold 1, with specific reactivity towards primary amines), and monomaleimido nanogold (Nanogold 2, with reactivity towards $S$-groups). Gold-OPH conjugates, dissolved in different buffers, were added at near molar ratios to the fluorescent decoy, and fluorescence intensity was measured and compared to fluorescence intensity of decoy alone. Measurements were taken as a function of time to ensure that equilibrium was reached. It was shown that after addition of OPH-nanogold the intensity of fluorescence increased significantly, suggesting that surface modified fluorescence was possible, and that enhancement of the fluorescence signal was achieved with both gold chemistries.

To evaluate the sensitivity of this approach, different paraoxon concentrations were added to the OPH-nanoparticle-decoy mixtures and a normalized ratio of fluorescence intensities (intensity of DDAO-Au-OPH in the presence of paraoxon compared to DDAO-Au-OPH in the absence of paraoxon) were measured. The greatest sensitivity to paraoxon (greatest slope) was obtained when decoy (DDAO) and $\mathrm{Au}-\mathrm{OPH}$ conjugate were present at near equimolar levels. In Fig. 7a and b, the representative fluorescence spectra of gold-OPH-decoy mixtures in the presence and absence of paraoxon are shown for the two different gold attachment chemistries. The large change in fluorescence intensity between DDAO and DDAO-Au-OPH when no paraoxon is present, which was observed with both types of gold-conjugates indicates that the fluorescence of the decoy (DDAO) is enhanced when the decoy is bound to the gold conjugate. The decrease in intensity of the DDAO-Au-OPH mixture when paraoxon was added is indicative of the 


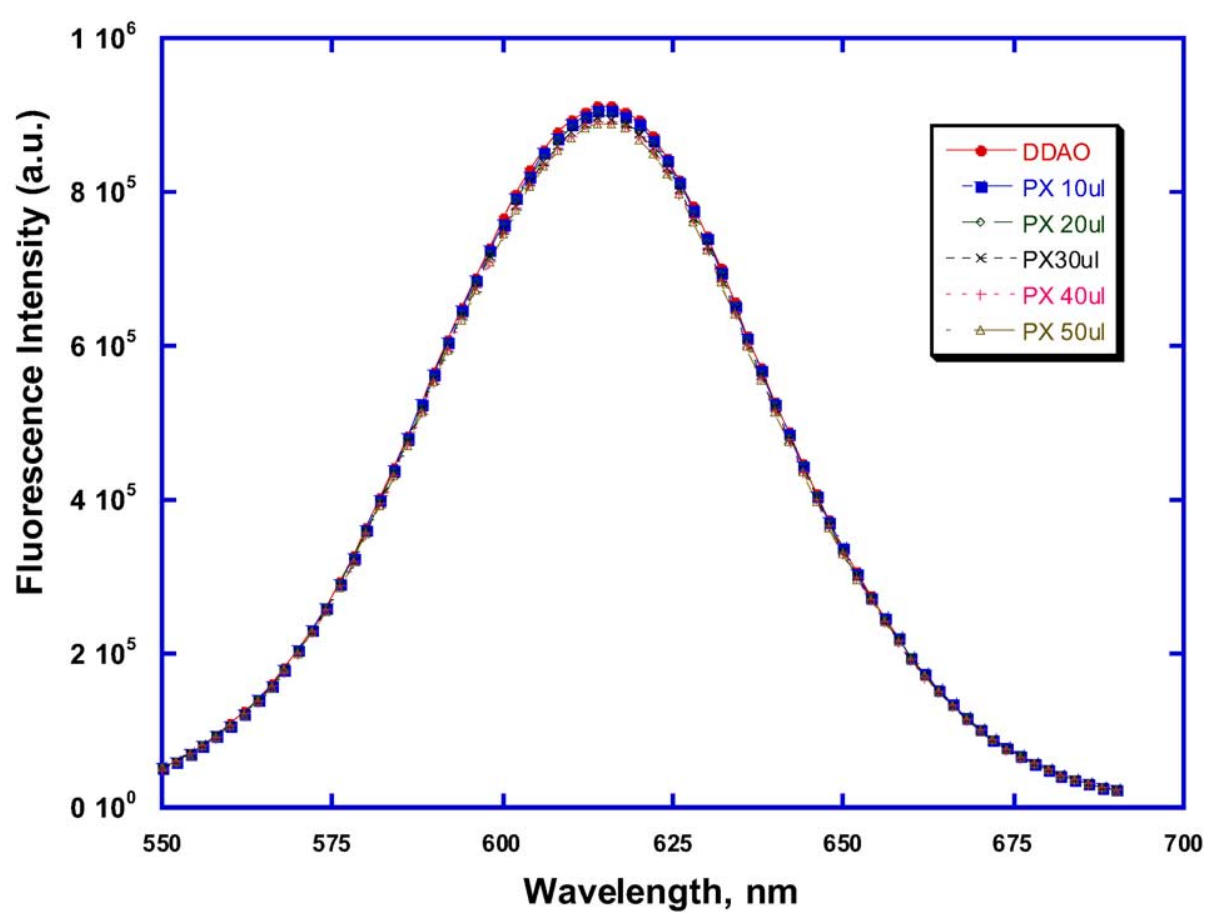

Fig. 6. Fluorescence of DDAO at varying paraoxon concentrations in the absence of OPH-gold complex. 20 mM Glycine buffer, pH 9.0. DDAO concentration $3 \times 10^{-6} \mathrm{M}$. Paraoxon (PX) concentration from $5 \times 10^{-6}$ to $2.5 \times 10^{-3} \mathrm{M}$; DDAO was excited at $478 \mathrm{~nm}$.

displacement of the DDAO by paraoxon. Both gold attachment chemistries produced conjugates which were capable of enhancing DDAO fluorescence upon binding. The greater enhancement in the absence of paraoxon observed in the conjugate prepared with the monomaleimido chemistry as compared to the sulfo- $N$-hydroxy-succinimido chemistry was probably due to the unique location of cysteine residues on the OPH as a point of attachment for the gold and differences in the average distance between gold and DDAO for the two chemistries. This is only conjecture as in no case was the actual distance between gold nanoparticle and enzyme active site measured experimentally.

A calibration curve for paraoxon is presented in Fig. 8. The minimum paraoxon concentration detected was $20 \mu \mathrm{M}$ which is near the $K_{M}$ of the enzyme for this substrate. Good linearity was observed at paraoxon concentrations up to $240 \mu$ M.

Based on these results, it is clear that nanoparticle-based sensing of OP substrates, using the catalytic power of an enzyme such as $\mathrm{OPH}$, is possible. An obvious advantage of this approach is that a $\mathrm{pH}$ measurement is excluded from the assay scheme. This makes analysis easier, since it is not necessary to work in weak buffer system. Another possible advantage of this type of sensor is the ability to control sensor performance via both $K_{M}$ of the enzyme for the OP compound of interest and the $K_{i}$ of the decoy used in the sensor. The relative magnitudes of $K_{M}$ and $K_{i}$ should strongly influence the slope of the calibration curve for the OP compound detected, and may provide a mechanism to discriminate between and/or identify different OP compounds in the environment. Finally, this type of detection method is not dependent upon the ability of the OPH enzyme to hydrolyze the OP compound, but only on the binding characteristics of the enzyme and OP. Thus, sensor performance will not be diminished for OP compounds weakly hydrolyzed by $\mathrm{OPH}$.

Sensor sensitivity and performance may be limited by fluorescence output of decoy and the affinity of decoy for OPH active site. A decoy with superior fluorescence output per molecule will increase the threshold of system sensitivity; however, a decoy with higher the quantum efficiency will exhibit a lower the change in fluorescence upon binding of the decoy to the nanoparticle-OPH conjugates. Thus there is clearly an optimum in fluorescence quantum efficiency for the decoy used.

Distance between gold nanoparticle and bound fluorophore greatly affect the fluorescence enhancement or fluorescence quenching possible in a system such as we describe. In these experiments, gold nanoparticle attachment was at any of the available binding sites on the surface, and was somewhat random. The presence of mutable different nanoparticle-enzyme attachments in solution led to a averaging of distance between decoy and nanoparticle, and probably led to a decreased fluorescence signal. This could be avoided by using single nanoparticle attachment site, which could be created by site specific mutation of the enzyme. In addition, size and surface roughness of the nanoparticle will influence the surface resonance effects responsible for fluorescence enhancement in this system.

Binding affinity of the decoy is the probably the most important parameter, outside of its fluorescence, that will 

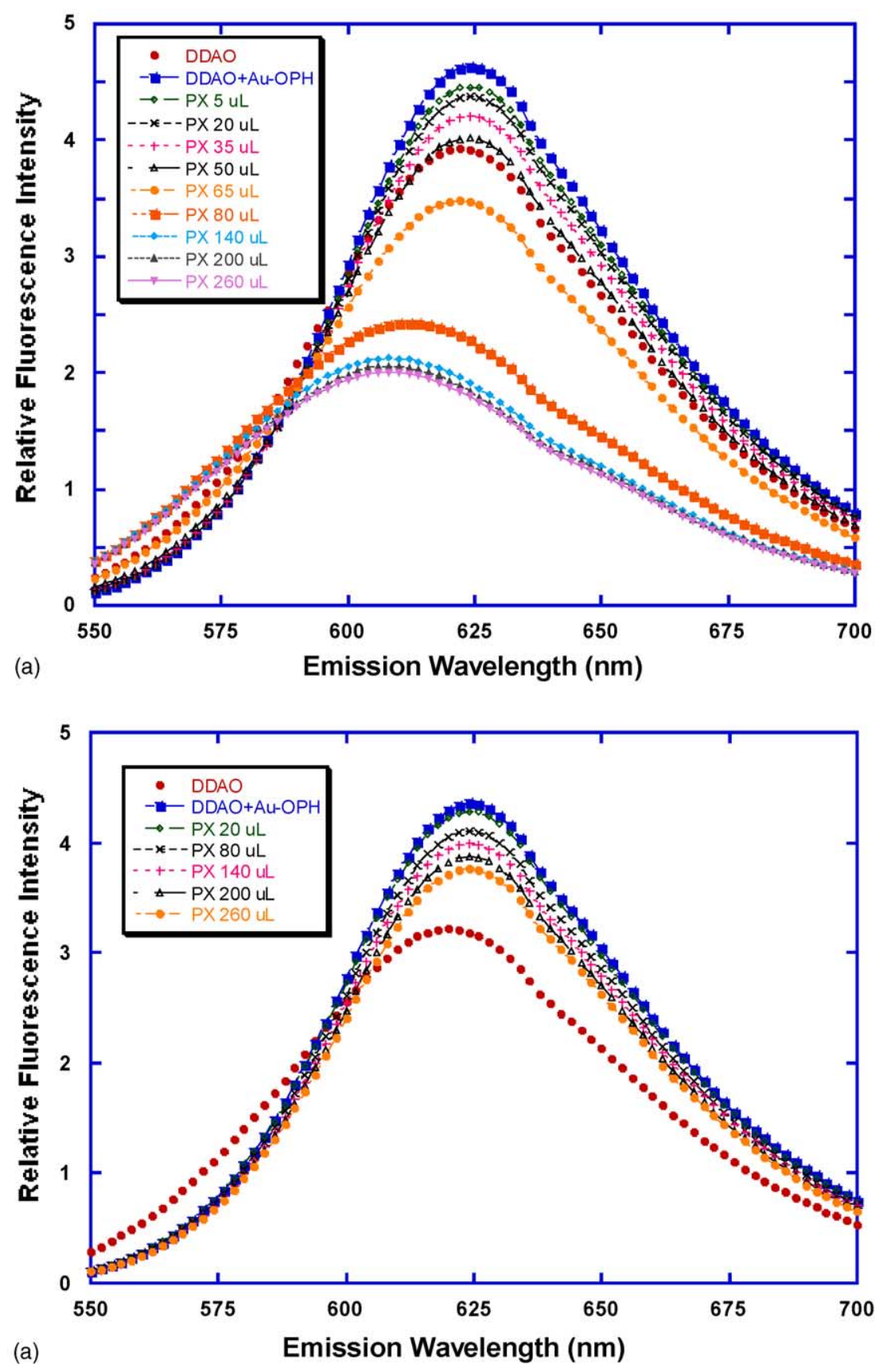

Fig. 7. System response on different paraoxon concentrations. Relative fluorescence intensity of gold-OPH-decoy system as a function of location/chemistry of gold nanoparticles attachment: (a) monomaleimido nanogold, (b) sulfo- $N$-hydroxy-succinimido nanogold. DDAO concentration $3 \times 10^{-6} \mathrm{M}$, Au-OPH conjugate: $40 \mu \mathrm{L}, 1 \mathrm{mg} / \mathrm{mL}$ in $\mathrm{OPH}, 1: 1 \mathrm{Au}-\mathrm{OPH}$ ratio. Paraoxon (PX) concentration from $5 \times 10^{-6}$ to $7.5 \times 10^{-3} \mathrm{M}$.

contribute to sensor performance. Molecules that are structurally similar to OP neurotoxins are likely to have the highest affinity for $\mathrm{OPH}$. While it is not straightforward to a priori design a decoy with a known affinity for an enzyme, it may be possible chemically alter decoys, i.e. prepare synthetic decoy with slight mismatches from an OPH substrate, which should yield decoys of different binding affinities. The better decoy should have the highest affinity for the OPH, but be displaceable by the desired OP substrate. Decoys with very high affinity for the $\mathrm{OPH}$ will require more $\mathrm{OP}$ substrate to be displaced, while decoys with low affinity for the $\mathrm{OPH}$ will lead to high background fluorescence and lower sensitivity. In addition, low affinity decoys may easily be displaced by other molecules, leading to loss of specificity of the sensor. There will exist an optimum in binding affinity of the decoy relative to the OP substrate for the OPH enzyme that yields the best sensor performance in terms of both sensitivity and selectivity. 


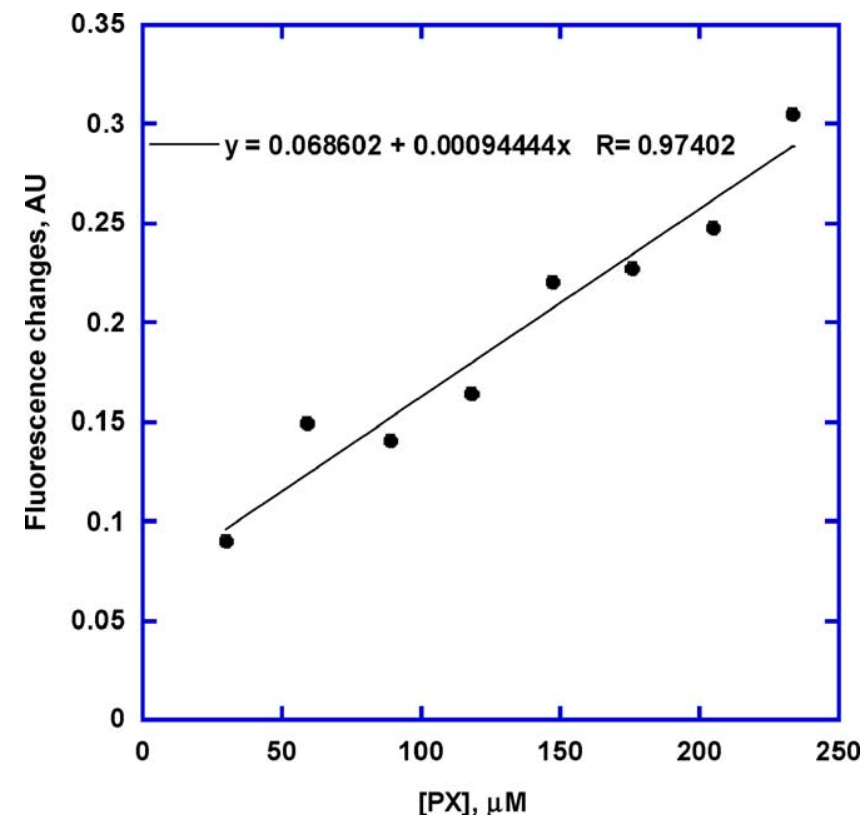

Fig. 8. Calibration curve. Relative fluorescence intensity change $(\Delta \mathrm{IF})$ is plotted as a function of added paraoxon (PX) concentration. A gold nanoparticles are sulfo- $N$-hydroxy-succinimido nanogold. $20 \mathrm{mM}$ Glycine buffer, $\mathrm{pH}$ 9.0, DDAO concentraton $3.0 \times 10^{-6} \mathrm{M}$, Au-OPH conjugate: $40 \mu \mathrm{L}$, $1 \mathrm{mg} / \mathrm{mL}$ in $\mathrm{OPH}, 1: 1 \mathrm{Au}-\mathrm{OPH}$ ratio.

As with other enzyme-based sensors, the performance of the method described will be affected by enzyme inactivation and by the presence of other competitive and non-competitive inhibitors of OPH in the environment. While the OPH used in these studies is relatively stable, calibration of the sensor will have to be performed to determine active enzyme levels prior to operation. As there is a possibility that other compounds in environmental samples that are not OP compounds may bind to OPH or otherwise alter the fluorescence of the decoy, in very sensitive applications, multiple sensing technologies should be used.

\section{Conclusion}

Results presented above show that nanoparticle-based sensing of enzyme substrates, using the catalytic power of an enzyme such as OPH is possible. An obvious advantage of this approach is that a $\mathrm{pH}$ measurement is excluded from the assay scheme that makes analysis easier. It may be possible to improve the sensitivity of the method by altering decoy characteristics, optimization of nanoparticles size and surface roughness, and site or distance specific attachment of the nanoparticle to the surface of enzyme molecule.

\section{Acknowledgments}

Support for this work comes from NSF Grant (CTS0330189 to ALS), US Army Medical Research and Materiel
Command (USAMRMC-C00-00243 to JRW) and from Auburn University Detection and Food Safety Center. The help of Melinda Wales, Janet Grimsley, Brian Kieintz, and Jared Wittry is gratefully acknowledged.

\section{References}

[1] P.T. Holland, C.P. Malcolm, Emerging Strategies for Pesticide Analysis, CRC Press, Boca Ration, 1992.

[2] D.P. Dumas, H.D. Durst, W.G. Landis, F.M. Raushel, J.R. Wild, Arch. Biochem. Biophys. 277 (1990) 155.

[3] G.A. Evtugyn, H.C. Budnikov, E.B. Nikolskaya, Analyst 121 (1996) 1911.

[4] N. Mionetto, J.-L. Marty, I. Karube, Biosens. Bioelectr. 9 (1994) 463.

[5] G. Palleschi, M. Bernabei, C. Cremisini, M. Mascini, Sens. Actuators B 7 (1992) 513.

[6] J.J. Kulys, E.J. D'Costa, Biosens. Bioelectron. 6 (1991) 109.

[7] A. Herbert, L. Guilhermino, H.C. Da Silva De Assis, P.-D. Hasen, Zeitschvift fur Angewande Zoologie 3 (1995/96) 1-15.

[8] M. Brufani, M. Marta, M. Pomponi, Eur. J. Biochem. 157 (1986) $115-120$.

[9] A.L. Simonian, E.I. Rainina, J.R. Wild, Anal. Lett. 30 (1997) 2453.

[10] A. Herbert, L. Guilhermino, H.C. Da Silva De Assis, P.-D. Hasen, Zeitschvift fur Angewande Zoologie. 3 (1995/96) 1.

[11] J.F. Payne, A. Mathieu, W. Melvin, L.L. Fancey, Marine Pollution Bulletin, 81

[12] T.T. Bachmann, R.D. Schmid, Anal Chim. Acta 401 (1999) 95.

[13] O. Schafer, L. Weil, R. Niessner, Methoden Vom Wasser 82 (1994) 233.

[14] E. Rainina, A. Simonian, A. Efremenko, S. Varfolomeyev, J. Wild Biosens, Bioelectronics 11 (1996) 991.

[15] C.S. McDaniel, L.L. Harper, J.R. Wild, J. Bacteriol. 170 (1988) 2306-2311.

[16] D.P. Dumas, J.R. Wild, F.M. Raushel, Biotech. Appl. Biochem. 11 (1989) 235.

[17] D.P. Dumas, H.D. Durst, W.G. Landis, F.M. Raushel, J.R. Wild, Arch. Biochem. Biophys. 277 (1990) 155.

[18] K. Lai, N.J. Stolowich, J.R. Wild, Arch. Biochem. Biophys. 318 (1995) 59.

[19] R. Russell, M. Pishko, A. Simonian, J. Wild, Anal. Chem. 71 (1999) 4909-4912.

[20] A.L. Simonian, B.D. diSioudi, J.R. Wild, Anal. Chim. Acta 389 (1999) 189-196.

[21] J. Lakowicz, I. Gryczynski, Z. Gryczynski, K. Nowaczyk, C. Murphy, Anal. Biochem. 280 (2000) 128-136.

[22] D. Feldheim, Optical and Electronic Properties of Metal Nanoparticle Arrays", 2001. http://www.ncsu.edu/chemistry/dlf/opticalprop.html.

[23] K. Sokolov, G. Chumanov, T.M. Cotton, J. Anal. Chem. (1998) 70

[24] W. Chan, S.M. Nie, Science 281 (5385) (1998) 2016-2018.

[25] T.A. Taton, C.A. Mirkin, R.L. Letsinger, Science 289 (2000) $1757-$ 1760.

[26] J.J. Storhoff, R. Elghanian, R.C. Mucic, C.A. Mirkin, R.L. Letsinger, J. Am. Chem. Soc. 120 (1998) 1959-1964.

[27] L.A. Luck, M.J. Moravan, J.E. Garland, B. Salopek-Sondi, D. Roy, Biosens. Bioelectron. 19 (2003) 249-259.

[28] E. Vogel, R. Geßner, M.H.B. Hayes, W. Kiefer, Characterisation of humic acid by means of SERS, J. Mol. Struct. 482-483 (1999) 195199.

[29] S. Cristoph, F.J. Meyer-Almes, Biopolymers (2003) 72.

[30] I.Y. Wang, Y.Y. Zhou, C.Q. Zhu, M.C. Mang, H.S. Tao, L. Wang, Chem. J. Chin. Univ. 24 (2003) 612-614.

[31] N. Lochner, F. Pittner, M. Wirth, F. Gabor, Pharm. Res. 20 (2003) 833-899. 
[32] H. Harma, A.M. Pelkkikangas, T. Soukka, P. Huhtinen, S. Huopalahti, T. Lovgren, Anal. Chim. Acta 482 (2003) 157-164.

[33] W. Chen, G. Martinez, A. Mulchandani, Anal. Biochem. 280 (2000) 166-172.

[34] D.J. Lichlyter, S.A. Grant, O. Soykan, Development of a novel FRET immunosensor technique, Biosens. Bioelectron. 19 (2003) 219-226.
[35] J. Kummerlen, A. Leitner, H. Brunner, F.R. Aussenegg, A. Wokaun, Mol. Phys. 80 (1993) 1031-1046.

[36] G. Bonnet, A. Libchaber, Optical sensitivity in molecular recognition, Physica A 263 (1999) 68-77.

[37] M.M. Benning, J.M. Kuo, F.M. Raushel, H.M. Holden, Biochemistry 33 (1994) 15001-15007. 